\title{
Hemorrhagic Shock Caused By Norovirus Infection In Henoch Schonlein Purpura: A Case Report
}

\author{
Authors: Valentina Juraga ${ }^{1}$, Saša Sršen ${ }^{2}$ (mentor) \\ ${ }^{1}$ School of Medicine, University of Zagreb, 10000 Zagreb, Republic of Croatia \\ ${ }^{2}$ Department of Pediatrics, Split University Hospital Centre, 21000 Split, Republic of Croatia \\ DOI: https://doi.org/10.26800/LV-142-supp5-5
}

\section{Background:}

Henoch Schonlein purpura (HSP) is the most common vasculitis in pediatric population. It's usually triggered by respiratory or gastrointestinal infections. HSP is characterized by a classic tetrad of nonthrombocytopenic palpable purpura, arthritis or arthralgias, gastrointestinal and renal involvement. Norovirus infection is generally self-limiting disease manifested by vomiting and diarrhoea, but when accompanied with HSP, it can cause some life treathening complications.

\section{Case report:}

A 12- year old girl presented with petechial rash on legs, spreading from feet to thighs bilaterally and with pain and swelling of the left knee and ankle. The physical examination also revealed pain and limited movement in the left radiocarpal joint, tachycardia and low-grade fever. A week before onset of symptoms, patient had a gastrointestinal infection and her fecal occult blood tests (FOBT) were positive and her stool tested positive for norovirus. Initial corticosteroid treatment led to improvement of her clinical appearance and negativisation of FOBT. Ten days after the admission she started vomiting and having bloody diarrhoea that quickly progressed to hematochezia. She was transferred to ICU in the state of compensated hypovolemic shock. Her abdominal CT scan showed distension of small intestine and thickening of both, colon and small intestine wall. After anti-hemorrhagic therapy her state improved significantly, she didn't have any gastrointestinal symptoms and therefore she was dissmised from the hospital.

\section{Discussion:}

More than $50 \%$ of patients with HSP have gastrointestinal symptoms that include abdominal pain, nausea, vomiting, hematemesis, melena, and hematochezia. These symtoms are result of mesenteric vasculitis, but in this case, additional factor in destruction of gastrointestinal wall was norovirus infection as it causes cytoplasmic vacuolization, and infiltration of polymorphonuclear and mononuclear cells into the lamina propria.

\section{Conclusion:}

This case report suggests thorough managment of HSP because when complicated with infections, such as this norovirus infection, it can lead to severe condition like hemorrhagic shock.

Keywords: Henoch-Schonlein purpura, norovirus, shock, vasculitis 